\title{
The Hopf algebra structure of multiple harmonic sums
}

\author{
Michael E. Hoffman ${ }^{\mathrm{a}}$

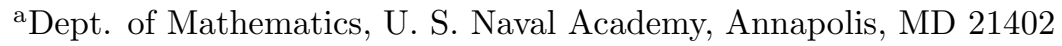

Multiple harmonic sums appear in the perturbative computation of various quantities of interest in quantum field theory. In this article we introduce a class of Hopf algebras that describe the structure of such sums, and develop some of their properties that can be exploited in calculations.

\section{MULTIPLE HARMONIC SUMS}

As discussed in the introduction of [1], multiple harmonic sums occur in perturbative higherorder calculations of quantum field theory. Let $I=\left(i_{1}, i_{2}, \ldots, i_{k}\right)$ be a sequence of positive integers. For positive integers $n$, we define the multiple harmonic sums

$$
\begin{aligned}
& A_{I}\left(n ; x_{1}, x_{2} \ldots, x_{k}\right)= \\
& \sum_{n \geq n_{1}>n_{2}>\cdots>n_{k} \geq 1} \frac{x_{1}^{n_{1}} x_{2}^{n_{2}} \cdots x_{k}^{n_{k}}}{n_{1}^{i_{1}} n_{2}^{i_{2}} \cdots n_{k}^{i_{k}}}
\end{aligned}
$$

and

$$
\begin{aligned}
& S_{I}\left(n ; x_{1}, x_{2} \ldots, x_{k}\right)= \\
& \sum_{n \geq n_{1} \geq n_{2} \geq \cdots \geq n_{k} \geq 1} \frac{x_{1}^{n_{1}} x_{2}^{n_{2}} \cdots x_{k}^{n_{k}}}{n_{1}^{i_{1}} n_{2}^{i_{2}} \cdots n_{k}^{i_{k}}}
\end{aligned}
$$

associated with $I$ (Note that the only difference between (11) and (2) is in the inequalities in the summation variables). Both types of sums appear in [11] and 13, with a slightly different notation ( $Z$ is used in place of our $A$ ).

If $i_{1}>1$ and $x_{1}=x_{2}=\cdots=x_{k}=1$, the sums (11) and (2) converge as $n \rightarrow \infty$, giving the well-known multiple zeta values 6 6147/15:

$$
\zeta\left(i_{1}, \ldots, i_{k}\right)=A_{\left(i_{1}, \ldots, i_{k}\right)}(\infty ; 1, \ldots, 1)
$$

which also occur in some perturbative QFT calculations 44. More generally, (11) and (2) converge as $n \rightarrow \infty$ when $\left|x_{i}\right|=1$ for all $i$ and $i_{1} x_{1} \neq 1$. The quantities

$$
\operatorname{Li}_{I}\left(x_{1}, \ldots, x_{n}\right)=A_{I}\left(\infty ; x_{1}, \ldots, x_{k}\right)
$$

are called multiple polylogarithms [2] they generalize the classical polylogarithm $\operatorname{Li}_{n}\left(x_{1}\right)=$ $A_{(n)}\left(\infty ; x_{1}\right)$.

It is immediate from the defining equations (11) and (2) that the sums $S_{I}$ can be written in terms of the $A_{I}$. To state the relation precisely, let $\mathcal{C}(n)$ be the set of compositions of $n$, i.e., ordered sequences $\left(i_{1}, \ldots, i_{k}\right)$ of positive integers with $i_{1}+\cdots+i_{k}=n$. If $I=\left(i_{1}, \ldots, i_{k}\right)$ is a composition of $n$ and $J=\left(j_{1}, \ldots, j_{p}\right)$ is a composition of $k$, then there is a composition $J \circ I$ of $n$ given by

$$
\begin{array}{r}
\left(i_{1}+\cdots+i_{j_{1}}, i_{j_{1}+1}+\cdots+i_{j_{1}+j_{2}}, \ldots,\right. \\
\left.i_{k-j_{p}+1}+\cdots+i_{k}\right)
\end{array}
$$

(cf. [8, p. 52]). Also, compositions act on argument strings: given $J=\left(j_{1}, \ldots, j_{p}\right) \in \mathcal{C}(k)$ and a string $X=\left(x_{1}, \ldots, x_{k}\right)$ of length $k$, we have

$$
\begin{aligned}
& J(X)= \\
& \quad\left(x_{1} \cdots x_{j_{1}}, x_{j_{1}+1} \cdots x_{j_{1}+j_{2}}, \ldots, x_{k-j_{p}+1} \cdots x_{k}\right) .
\end{aligned}
$$

Then the relation between sums of types (II) and (21) is given by

$S_{I}(n ; X)=\sum_{J \in \mathcal{C}(k)} A_{J \circ I}(n ; J(X))$

for any $I=\left(i_{1}, \ldots, i_{k}\right)$ and $X=\left(x_{1}, \ldots, x_{k}\right)$. Möbius inversion can be applied to (3) to obtain

$$
A_{I}(n ; X)=\sum_{J \in \mathcal{C}(k)}(-1)^{\ell(J)-k} S_{J \circ I}(n ; J(X)),
$$

where $\ell(J)$ is the number of parts of $J$. In fact, there is a deeper relation between the $S_{I}$ and the 
$A_{I}$ than the essentially trivial equations (3) and (4): the two types of sums are dual in the sense that they have, up to signs, the same algebraic properties. In the case where the arguments $x_{i}$ are roots of unity, we can formalize the algebra of such sums using the family of Hopf algebras described in the next section. (Making the arguments roots of unity seems to capture many cases of physical interest: see, e.g., [3].)

\section{THE EULER ALGEBRA}

We recall from [8] the construction of the Euler algebra of index $r$, where $r$ is a positive integer. We start with noncommuting symbols (or "letters") $z_{i, j}$, where $i, j$ are integers with $i$ positive and $0 \leq j \leq r-1$. Let $\mathcal{E}_{r}$ be the complex vector space generated by words in the $z_{i, j}$ (including the empty word, denoted by 1 ). For such a word $w=z_{i_{1}, j_{1}} z_{i_{2}, j_{2}} \cdots z_{i_{k}, j_{k}}$, we define the degree of $w$ to be $|w|=i_{1}+\cdots+i_{k}$ (and call $\ell(w)=k$ the length of $w$.) Now we define a multiplication $*$ on $\mathcal{E}_{r}$ as follows. We require $1 * w=w * 1=w$ for all words $w$, and

$$
\begin{aligned}
& z_{i, j} w_{1} * z_{m, n} w_{2}=z_{i, j}\left(w_{1} * z_{m, n} w_{2}\right)+ \\
& \quad z_{m, n}\left(z_{i, j} w_{1} * w_{2}\right)+z_{i+m, j+n}\left(w_{1} * w_{2}\right)
\end{aligned}
$$

for any words $w_{1}, w_{2}$ : here the addition in the second subscript is to be understood $\bmod r$. For example, when $r=3$

$$
\begin{gathered}
z_{1,1} * z_{1,2} z_{2,1}=z_{1,1} z_{1,2} z_{2,1}+z_{1,2}\left(z_{1,1} * z_{2,1}\right)+z_{2,0} z_{2,1} \\
=z_{1,1} z_{1,2} z_{2,1}+z_{1,2} z_{1,1} z_{2,1}+z_{1,2} z_{2,1} z_{1,1}+ \\
z_{1,2} z_{3,2}+z_{2,0} z_{2,1} .
\end{gathered}
$$

Since each of the parenthesized products on the right-hand side of (5) has total length less than the left-hand side, equation (15) gives an inductive definition of $*$ on $\mathcal{E}_{r}$. As shown in $\left[8,\left(\mathcal{E}_{r}, *\right)\right.$ is a commutative, associative graded algebra over C. In fact, $\left(\mathcal{E}_{r}, *\right)$ is a polynomial algebra. To describe the generators, we first assume that the letters $z_{i, j}$ are totally ordered, and extend this order lexicographically to words. A word $w$ is called Lyndon if it is smaller than any of its proper right factors $(v \neq 1$ is a proper right factor of $w$ if $w=u v$ for $u \neq 1$ ). Then we have the following result [8, Theorem 2.6].
Theorem 2.1. For positive integer $r,\left(\mathcal{E}_{r}, *\right)$ is the polynomial algebra on the Lyndon words.

Remark. From the discussion in [8, Example 2], the number of Lyndon words of degree $n$ in $\mathcal{E}_{r}$ is

$$
\frac{1}{n} \sum_{d \mid n} \mu\left(\frac{n}{d}\right)(r+1)^{d},
$$

where the sum is over divisors of $n$ and $\mu$ is the Möbius function on the integers.

In the case $r=1,\left(\mathcal{E}_{r}, *\right)$ is the algebra QSym of quasi-symmetric functions as defined by Gessel [5]. For a description of QSym and its relation to multiple harmonic sums with unit arguments, see 9. Note that QSym contains the well-known algebra Sym of symmetric functions: in fact, Sym can be imbedded in any $\mathcal{E}_{r}$ by sending the elementary symmetric function $e_{i}$ to $z_{1,0}^{i}$.

We can also define a coalgebra structure on $\mathcal{E}_{r}$ as follows. The counit $\epsilon: \mathcal{E}_{r} \rightarrow \mathbb{C}$ is given by

$$
\epsilon(1)=1, \quad \epsilon(w)=0 \quad \text { for }|w|>0
$$

and the coproduct $\Delta: \mathcal{E}_{r} \rightarrow \mathcal{E}_{r} \otimes \mathcal{E}_{r}$ by

$$
\begin{aligned}
& \Delta\left(z_{i_{1}, j_{1}} z_{i_{2}, j_{2}} \cdots z_{i_{k}, j_{k}}\right)= \\
& \quad \sum_{p=0}^{k} z_{i_{1}, j_{1}} \cdots z_{i_{p}, j_{p}} \otimes z_{i_{p+1}, j_{p+1}} \cdots z_{i_{k}, j_{k}} .
\end{aligned}
$$

Then Theorem 3.1 of $[8]$ says that $\left(\mathcal{E}_{r}, *, \Delta\right)$ is a graded connected Hopf algebra.

Since the $*$-product is commutative, the antipode $S$ of $\left(\mathcal{E}_{r}, *, \Delta\right)$ is an involution, i.e., an algebra automorphism with $S^{2}=$ id (see, e.g., 10 Theorem III.3.4]). As shown in [8 Theorem 3.2], there are two (not obviously identical) formulas for $S$. Our first formula for $S$ involves iterated products:

$S(w)=\sum_{w_{1} w_{2} \cdots w_{k}=w}(-1)^{k} w_{1} * w_{2} * \cdots * w_{k}$,

where the sum is over all decompositions of $w$ into (nonempty) subwords $w_{1}, \ldots, w_{k}$.

For the second formula, we shall introduce some more notation. Given a string $a_{1}, \ldots, a_{n}$ of letters, let $\left[a_{1}, \ldots, a_{n}\right]$ be the letter obtained 
by adding all the subscripts (where of course the addition in the second subscript is $\bmod r$ ). Then the multiplication rule (5) can be written

$$
\begin{aligned}
& a w * b v= \\
& \quad a(w * b v)+b(a w * v)+[a, b](w * v)
\end{aligned}
$$

for letters $a, b$ and words $w, v$. As above, let $\mathcal{C}(n)$ be the set of compositions of $n$. Then $\left(i_{1}, \ldots, i_{k}\right) \in \mathcal{C}(n)$ acts on a word $w=a_{1} \cdots a_{n}$ of length $n$ as follows:

$$
\begin{aligned}
& \left(i_{1}, \ldots, i_{k}\right)[w]= \\
& {\left[a_{1}, \ldots, a_{i_{1}}\right]\left[a_{i_{1}+1}, \ldots, a_{i_{1}+i_{2}}\right] \cdots\left[a_{n-i_{k}+1}, \ldots, a_{n}\right] .}
\end{aligned}
$$

Our second formula for the antipode can be written in terms of this action:

$S(w)=(-1)^{n} \sum_{I \in \mathcal{C}(n)} I\left[a_{n} a_{n-1} \cdots a_{1}\right]$

for words $w=a_{1} \cdots a_{n}$ of length $n$.

Now let $R: \mathcal{E}_{r} \rightarrow \mathcal{E}_{r}$ be the linear function that reverses words, i.e.,

$$
R\left(a_{1} a_{2} \cdots a_{n}\right)=a_{n} \cdots a_{2} a_{1} .
$$

The following result can be proved by induction on word length (see [15] Theorem 9]).

Theorem 2.2. The function $R: \mathcal{E}_{r} \rightarrow \mathcal{E}_{r}$ is a *-automorphism.

Since clearly $\Delta \circ R=(R \otimes R) \circ \Delta, R$ is evidently an automorphism of the Hopf algebra $\left(\mathcal{E}_{r}, *, \Delta\right)$.

The action of compositions on words of $\mathcal{E}_{r}$ can be used to put a partial order $\preceq$ on words as follows. For a word $w=a_{1} \cdots a_{n}$ of length $n$, set $v \preceq w$ if $v=I[w]$ for some $I \in \mathcal{C}(n)$ (Note that in this case $\ell(v) \leq \ell(w)$ and $|v|=|w|)$. Define

$\bar{w}=\sum_{v \preceq w} v=\sum_{J \in \mathcal{C}(\ell(w))} J[w]$

for words $w$ of $\mathcal{E}_{r}$.

Our second formula for the antipode can now be written

$$
R S(w)=S R(w)=(-1)^{\ell(w)} \bar{w}
$$

for any word $w$ of $\mathcal{E}_{r}$. Equating the two formulas for the antipode, we have

$$
\begin{aligned}
& \sum_{w_{1} w_{2} \cdots w_{k}=w}(-1)^{k} w_{1} * w_{2} * \cdots * w_{k}= \\
&(-1)^{\ell(w)} \sum_{v \preceq w} R(v),
\end{aligned}
$$

or, after applying $R$ to both sides,

$\bar{w}=\sum_{w_{1} \cdots w_{k}=R(w)}(-1)^{\ell(w)-k} w_{1} * \cdots * w_{k}$.

Now apply $R S$ to both sides of equation (10):

$$
w=\sum_{w_{1} \cdots w_{k}=R(w)}(-1)^{\ell(w)-k} \bar{w}_{1} * \cdots * \bar{w}_{k} .
$$

Also, since $\bar{w}=(-1)^{\ell(w)} S R(w)$ and $S R$ is an automorphism of the Hopf algebra $\left(\mathcal{E}_{r}, *, \Delta\right)$, we can work with the vector space basis $\bar{w}$ just as well as with the basis consisting of the words $w$ : the only difference is that the inductive rule (17) for the $*$-product is replaced by

$$
\overline{a w} * \overline{b v}=\overline{a(w * b v)}+\overline{b(a w * v)}-\overline{[a, b](w * v)} .
$$

\section{RELATION TO MULTIPLE HAR- MONIC SUMS}

Now we relate the Hopf algebras $\mathcal{E}_{r}$ to the multiple harmonic sums. For fixed $n$, define a linear $\operatorname{map} \rho_{n}: \mathcal{E}_{r} \rightarrow \mathbb{C}$ by

$$
\rho_{n}\left(z_{i_{1}, j_{1}} \cdots z_{i_{k}, j_{k}}\right)=A_{\left(i_{1}, \ldots, i_{k}\right)}\left(n ; \epsilon^{j_{1}}, \ldots, \epsilon^{j_{k}}\right)
$$

where $\epsilon=e^{\frac{2 \pi i}{r}}$. We have the following result.

Theorem 3.1. The function $\rho_{n}$ is a homomorphism of $\left(\mathcal{E}_{r}, *\right)$ into $\mathbb{C}$.

Proof. $\rho_{n}$ is the composition of the homomorphism $\phi_{n}: \mathcal{E}_{r} \rightarrow \mathbb{C}\left[t_{1}, \ldots, t_{n}\right]$ of [8. Theorem 7.1] with the homomorphism $\mathbb{C}\left[t_{1} \ldots, t_{n}\right] \rightarrow \mathbb{C}$ sending $t_{i}$ to $1 / i, 1 \leq i \leq n$.

Comparing the definition (9) of $\bar{w}$ with equation (3), it is evident that

$$
\rho_{n}\left(\overline{z_{i_{1}, j_{1}} \cdots z_{i_{k}, j_{k}}}\right)=S_{\left(i_{1}, \ldots, i_{k}\right)}\left(n ; \epsilon^{j_{1}}, \ldots, \epsilon^{j_{k}}\right) .
$$


Henceforth we shall write $A_{w}(n)$ for $\rho_{n}(w)$ and $S_{w}(n)$ for $\rho_{n}(\bar{w})$ for words $w$ of $\mathcal{E}_{r}$, using the word to code for both the exponents and roots-of-unity arguments. In this notation, equation (3) is

$S_{w}(n)=\sum_{u \preceq w} A_{u}(n)$.

Now applying $\rho_{n}$ to equations (10) and (11) gives respectively

$$
\begin{aligned}
& S_{w}(n)= \\
& \sum_{w_{1} \cdots w_{k}=R(w)}(-1)^{\ell(w)-k} A_{w_{1}}(n) \cdots A_{w_{k}}(n)
\end{aligned}
$$

and

$$
\begin{aligned}
& A_{w}(n)= \\
& \sum_{w_{1} \cdots w_{k}=R(w)}(-1)^{\ell(w)-k} S_{w_{1}}(n) \cdots S_{w_{k}}(n) .
\end{aligned}
$$

By equating the right-hand sides of equations (12) and (13), one obtains

$$
\begin{aligned}
& \sum_{\substack{A_{1} \\
w_{1} \cdots w_{k}=R(w) \\
k>1}}(-1)^{\ell(w)-k} A_{w_{1}}(n) \cdots A_{w_{k}}(n) \\
& \quad-\sum_{u \prec w} A_{u}(n),
\end{aligned}
$$

which shows that $A_{w}(n)+(-1)^{\ell(w)} A_{R(w)}(n)$ can always be written in terms of sums of length less than than $\ell(w)$. Cf. the discussion in [13, $\S 6]$.

\section{EXAMPLE: SYMMETRIC SUMS}

To illustrate the use of the techniques introduced above, we show how symmetric linear combinations of the $A_{w}$ and $S_{w}$ can be written in terms of ordinary (length 1 ) harmonic sums. We start in $\mathcal{E}_{r}$. Note that the symmetric group $\Sigma_{k}$ acts on words of $k$ letters by permutation, i.e.,

$$
\sigma \cdot a_{1} \cdots a_{k}=a_{\sigma^{-1}(1)} \cdots a_{\sigma^{-1}(k)} .
$$

Fix a word $w=a_{1} a_{2} \cdots a_{k}$ of $\mathcal{E}_{r}$, and for a set partition $\mathcal{C}=\left\{C_{1}, \ldots, C_{p}\right\}$ of $\{1,2, \ldots, k\}$ let

$\mathcal{C}(w)=\left[a_{i}, i \in C_{1}\right] *\left[a_{i}, i \in C_{2}\right] * \cdots *\left[a_{i}, i \in \mathcal{C}_{p}\right]$.
Then repeated use of the multiplication rule (7) allows $\mathcal{C}(w)$ to be written as

$$
\sum_{\left\{B_{1}, \ldots, B_{q}\right\} \preceq \mathcal{C}} \sum_{\sigma \in \Sigma_{q}} \sigma \cdot\left[a_{i}, i \in B_{1}\right] \cdots\left[a_{i}, i \in B_{q}\right],
$$

where $\preceq$ is the partial order given by refinement. Now Möbius inversion can be applied to give

$$
\begin{aligned}
\sum_{\sigma \in \Sigma_{p}} \sigma \cdot\left[a_{i}, i \in C_{1}\right] \cdots\left[a_{i}, i \in C_{p}\right]= & \sum_{\mathcal{B}=\left\{B_{1}, \ldots, B_{q}\right\} \preceq \mathcal{C}} \mu(\mathcal{B}, \mathcal{C}) \mathcal{B}(w)
\end{aligned}
$$

where $\mu$ is the Möbius function for the partially ordered set of partitions of $\{1,2, \ldots, k\}$. When $\mathcal{C}=\{\{1\},\{2\}, \ldots,\{k\}\}$, then $\mu(\mathcal{B}, \mathcal{C})=c(\mathcal{B})$, where

$$
c(\mathcal{B})=(-1)^{k-q}\left(\operatorname{card} B_{1}-1\right) ! \cdots\left(\operatorname{card} B_{q}-1\right) !
$$

(see Example 3.10.4 of [12]). In this case equation (15) is

$$
\begin{aligned}
& \sum_{\sigma \in \Sigma_{k}} \sigma \cdot w= \\
& \sum_{\mathcal{B}=\left\{B_{1}, \ldots, B_{q}\right\}} c(\mathcal{B})\left[a_{i}, i \in B_{1}\right] * \cdots *\left[a_{i}, i \in B_{q}\right],
\end{aligned}
$$

where the sum on the right-hand side is over all partitions $\mathcal{B}$ of $\{1, \ldots, k\}$. Now apply $R S$ to both sides of equation (16) (and cancel signs) to get

$$
\begin{aligned}
& \sum_{\sigma \in \Sigma_{k}} \sigma \cdot \bar{w}= \\
& \sum_{\mathcal{B}=\left\{B_{1}, \ldots, B_{q}\right\}}|c(\mathcal{B})|\left[a_{i}, i \in B_{1}\right] * \cdots *\left[a_{i}, i \in B_{q}\right] .
\end{aligned}
$$

Finally, we can apply the homomorphism $\rho_{n}$ to equations (16) and (17) to get formulas for symmetric combinations of multiple harmonic sums in terms of ordinary harmonic sums:

$$
\begin{aligned}
\sum_{\sigma \in \Sigma_{k}} A_{\sigma \cdot w}(n)= & \\
& \sum_{\mathcal{B}=\left\{B_{1}, \ldots, B_{q}\right\}} c(\mathcal{B}) A_{\left[a_{i}, i \in B_{1}\right]}(n) \cdots A_{\left[a_{i}, i \in B_{q}\right]}(n)
\end{aligned}
$$




$$
\begin{aligned}
& \sum_{\sigma \in \Sigma_{k}} S_{\sigma \cdot w}(n)= \\
& \sum_{\mathcal{B}=\left\{B_{1}, \ldots, B_{q}\right\}}|c(\mathcal{B})| A_{\left[a_{i}, i \in B_{1}\right]}(n) \cdots A_{\left[a_{i}, i \in B_{q}\right]}(n) .
\end{aligned}
$$

Equations (18) and (19) generalize Theorem 4.1 of [9] (which is the case $r=1$ ). They may be compared to the corresponding formulas for multiple zeta values, which appear as Theorems 2.2 and 2.1, respectively, of [6. Equation (19) should also be compared to equations (2.37-2.41) of [1], which exhibit the cases $k=2, \ldots, 6$ for $r=2$.

In the special case $w=a^{k}$ (i.e., $w$ is a power of a single letter), equation (19) reduces to

$$
\begin{aligned}
& S_{a^{k}}(n)= \\
& \frac{1}{k !} \sum_{\mathcal{B}=\left\{B_{1}, \ldots, B_{q}\right\}}|c(\mathcal{B})| A_{\left[b_{1} a\right]}(n) \cdots A_{\left[b_{q} a\right]}(n),
\end{aligned}
$$

where $b_{i}=\operatorname{card} B_{i}$ and $[k a]$ means $[a, a, \ldots, a]$ with $k$ repetitions of $a$. Now for a given (unordered) sequence of positive integers $b_{1}, \ldots, b_{q}$ adding up to $k$, there are

$$
\frac{1}{m_{1} ! \cdots m_{k} !} \frac{k !}{b_{1} ! \cdots b_{q} !}
$$

partitions of the set $\{1,2 \ldots, k\}$ having the $b_{i}$ as block sizes, where $m_{s}=\operatorname{card}\left\{b_{i} \mid b_{i}=s\right\}$. Thus, equation (20) can be written as

$$
\begin{aligned}
& S_{a^{k}}(n)= \\
& \sum_{b_{1}+\cdots+b_{q}=k} \frac{1}{m_{1} ! \cdots m_{k} !} \frac{1}{b_{1}} A_{\left[b_{1} a\right]}(n) \cdots \frac{1}{b_{q}} A_{\left[b_{q} a\right]}(n),
\end{aligned}
$$

where the sum is over all integer partitions of $k$ (cf. equations (2.42-2.46) of [1]). There is an analogous formula for $A_{a^{k}}(n)$ differing from (21) only in the presence of signs.

\section{REFERENCES}

1. J. Blümlein, Algebraic relations between harmonic sums and associated quantities, Comput. Phys. Commun. 159 (2004), 19-54; preprint hep-ph/031106.
2. D. Bowman and D. Bradley, Multiple polylogarithms: a brief survey, in $q$-Series with $A p$ plications to Combinatorics, Number Theory, and Physics, Contemp. Math. 291, Amer. Math. Soc., Providence, 2001, pp. 71-92; preprint math.CA/0310062

3. D. J. Broadhurst, Massive 3-loop Feynman diagrams reducible to $\mathrm{SC}^{*}$ primitives of algebras at the sixth root of unity, Eur. Phys. J. C Part. Fields 8 (1999), 311-333; preprint hep-th/9803091

4. D. J. Broadhurst and D. Kreimer, Association of multiple zeta values with positive knots via Feynman diagrams up to 9 loops, Phys. Lett. B 393 (1997), 403-412; preprint hep-th/9609128

5. I. M. Gessel, Multipartite P-partitions and inner products of skew Schur functions, in Combinatorics and Algebra, Contemp. Math. 34, Amer. Math. Soc., Providence, 1984, pp. 289301.

6. M. E. Hoffman, Multiple harmonic series, $P a$ cific J. Math. 152 (1992), 275-290.

7. M. E. Hoffman, The algebra of multiple harmonic series, J. Algebra 194 (1997), 477-495.

8. M. E. Hoffman, Quasi-shuffle products, J. Algebraic Combin. 11 (2000), 49-68; preprint math.QA/9907173.

9. M. E. Hoffman, Quasi-symmetric functions and $\bmod p$ multiple harmonic sums, preprint math.NT/0401319.

10. C. Kassel, Quantum Groups, Springer-Verlag, New York, 1995.

11. S. Moch, P. Uwer and S. Weinzierl, Nested sums, expansions of transcendental functions and multi-scale multi-loop integrals, $J$. Math. Phys. 43 (2002), 3363-3386; preprint hep-ph/0110083

12. R. P. Stanley, Enumerative Combinatorics, vol. 1, Cambridge University Press, New York, 1997.

13. S. Weinzierl, Algebraic algorithms in perturbative calculations, preprint hep-th/0305260

14. D. Zagier, Values of zeta functions and their applications, in First European Congress of Mathematics, vol. 2, Birkhauser Boston, Cambridge, Mass., 1994, pp. 497-512. 
15. W. Zudilin, Algebraic relations for multiple zeta values, Russian Math. Surv. 58 (2003), 1-29. 